\title{
The Hot Path SSA Form: Extending the Static Single Assignment Form for Speculative Optimizations
}

\author{
Subhajit Roy and Y.N. Srikant \\ Computer Science and Automation Department, \\ Indian Institute of Science \\ \{subhajit,srikant\}@csa.iisc.ernet.in
}

\begin{abstract}
The Static Single Assignment (SSA) form has been an eminent contribution towards analyzing programs for compiler optimizations. It has been affable to the design of simpler algorithms for existing optimizations, and has facilitated the development of new ones. However, speculative optimizations - optimizations targeted towards speeding-up the "common cases" of a program — have not been fortunate enough to savor an SSA-like intermediate form. We extend the SSA form for speculative analyses and optimizations by allowing only hot reaching definitions - definitions along frequent acyclic paths in the program profile - to reach its respective uses; we call this representation the Hot Path $S S A$ form. We propose an algorithm for constructing such a form, and demonstrate its effectiveness by designing the analysis phase of a novel optimization - Speculative Sparse Conditional Constant Propagation: an almost obvious extension of Wegman and Zadeck's Sparse Conditional Constant Propagation algorithm. Our experiments on some SPEC2000 programs proves the potency of such an optimization.
\end{abstract}

\section{Introduction}

Program analyses and optimizations have benefited immensely from the SSA form as an intermediate representation. An extremely simple idea - allow only a single definition of a variable to reach the statements using it - prunes out false dependencies, and factors long use-def chains into a web of short, simple ones. A multitude of optimizations were either made possible, or were heavily empowered by the SSA form - sparse conditional constant propagation, global value numbering, and strength reduction to name a few.

However, speculative optimizations - optimizations biased towards frequently executed paths — have not been fortunate enough to enjoy an SSA-like intermediate representation. These optimizations have recently attracted a lot of attention, and are now recognised as a major vehicle towards improving program performance.

Modern compilation systems, acknowledging the importance of such unconventional optimizations, have started providing support for speculative analysis and transformation. However, in most of the intermediate representations, 
the profiling information is not integrated into the static program representation. This makes implementing speculative optimizations cumbersome, having to handle too many data-structures. Additionally, the absence of an SSA-like sparse representation has hindered the development of efficient algorithms for speculative optimizations.

We propose to extend the power of the SSA form to speculative optimizations by separating the hot use-def chains from the cold ones, thus allowing a speculative optimizer to "see" only the most-likely dataflow facts. However, the "non-speculative" SSA form is not lost: a traditional optimizer can still choose to constrain itself to the non-speculative form by ignoring the speculative information. The SSA form is not erased — just suitably extended with speculative information - obviating the necessity of constructing and maintaining the nonspeculative SSA form separately; at the same time, this SSA-like intermediate form is much more amenable to speculative analyses and optimizations.

We call this extension to the SSA form as the "Hot Path SSA (HPSSA) form". As the HPSSA form honours the constraint imposed by the SSA form (that of a single reaching definition for every use), many of the SSA-based algorithms for traditional optimizations developed over the last couple of decades (almost) immediately become available to speculative optimizers.

Following are our contributions in this paper:

- We propose a novel program representation - the Hot Path SSA (HPSSA) form - that allows a use to witness only the "more-likely" reaching definitions (section 4);

- We present an algorithm for constructing the HPSSA form (section 5);

- We demonstrate the potency of the HPSSA form by designing the analysis phase of a novel speculative optimization — Speculative Sparse Conditional Constant Propagation (SSCP) — that identifies both "safe" (expressions that are sure to be constants) and "speculative" (expressions that are more-likely to be constants) constants in a given program. An almost trivial extension of Wegman and Zadeck's SCP algorithm [21, SSCP exhibits the possibilities of developing new speculative optimizations using the HPSSA form by tailoring of existing SSA-based traditional optimizations (section 6).

\section{Background}

\subsection{The Static Single Assignment Form}

A program is said to be in Static Single Assignment (SSA) form if each use of a variable has exactly one reaching definition. A special operator, the $\phi$-function, merges multiple definitions from different paths into a single definition, forcing any subsequent use to see exactly one definition.

Figure 1 shows the SSA form of a program. Notice how the definitions of $x$ at $b_{1}, d_{1}$ and $e_{1}$ are "merged" into a single definition at the statement $f_{1}$, thus making $x_{9}$ the only definition reaching the uses $g_{3}, h_{3}$ and $i_{1}$. Understandably, the use-def structure of a program in SSA form is extremely simple - allowing the design of cleaner and faster algorithms. 


\subsection{Acyclic Path Profiling}

Ball and Larus 2] proposed an efficient algorithm for profiling acyclic paths - paths that terminate either at loopbackedges or at procedure exits. Essentially, an acyclic path profiler "chops-off" paths at a backedge, erasing the sequence in which the acyclic paths in the loop were actually executed. The Ball-Larus algorithm is widely used for speculative analyses and optimizations. We use acyclic path profiles to expose frequent use-def chains in the HPSSA form.

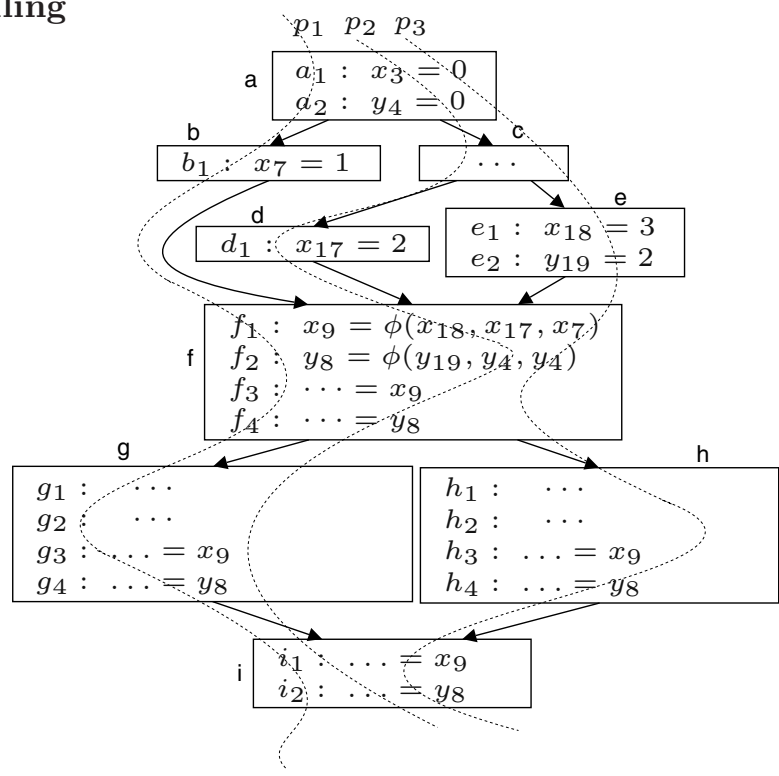

Fig. 1. A program in the SSA form. (Hot acyclic paths: $p_{1}$ :abfgi; $p_{2}$ :acdfgi; $p_{3}$ :acefhi).

\subsection{A Peek at the Hot Path SSA Form}

In this paper, we propose to tie the run-time behaviour of a program - as indicated by the frequently executed acyclic paths - directly to its static program representation, thus providing a convenient data-structure for the speculative optimizers. In the proposed representation, which we call the Hot Path SSA (HPSSA) form, an additional construct - the $\tau$-function - is introduced to capture information relevant for speculative analyses and optimizations. The $\tau$-functions act as "filters", separating the more-likely use-def chains from the lesser-likely ones. The first argument of the $\tau$-function is the traditional meetover-all-paths reaching definition; the rest of the arguments are the "hot" reaching definitions: definitions that are more-likely to reach the respective program point.

Figure 2 shows the HPSSA form of the program in Figure 1 Consider the basic-block $g$ : the $\tau$-function at $g_{1}$ indicates that $x_{9}$ is the "safe" meet-of-allpaths reaching definition, though the definitions of $x_{7}$ and $x_{17}$ are more likely to reach this program point (via the $\phi$-statement at $f_{1}$ ). Similarly, for $g_{4}, h_{3}$ and $h_{4}$, the hot reaching definitions are from definitions of $y_{4}, x_{18}$ and $x_{19}$ respectively - all of which are definitions to constants. Hence, the HPSSA form exposes the fact that the variables $y_{12}, x_{14}$ and $y_{15}$ are more likely to be constants with values 0,3 and 2 respectively - enabling a speculative optimizer to speculatively "predict" the value of these variables.

Though the HPSSA form uses acyclic path profiles, it is still adept at propagating hot reaching definitions across loop-boundaries. Figure 3 shows the 


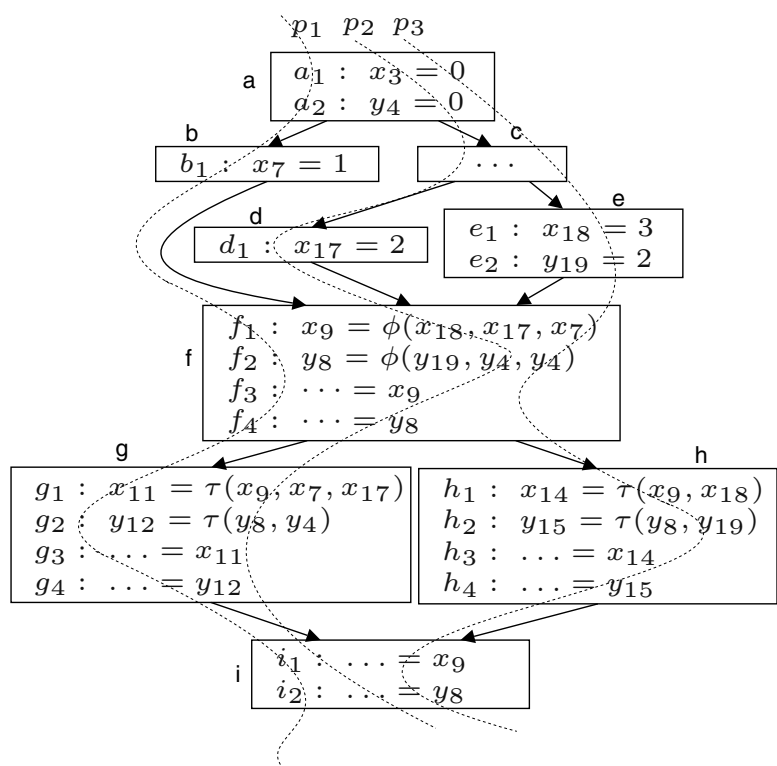

Fig. 2. The program in Figure 1 translated to the Hot Path SSA (HPSSA) form (Hot paths: $p_{1}$ :abfgi; $p_{2}$ :acdfgi; $p_{3}$ :acefhi)

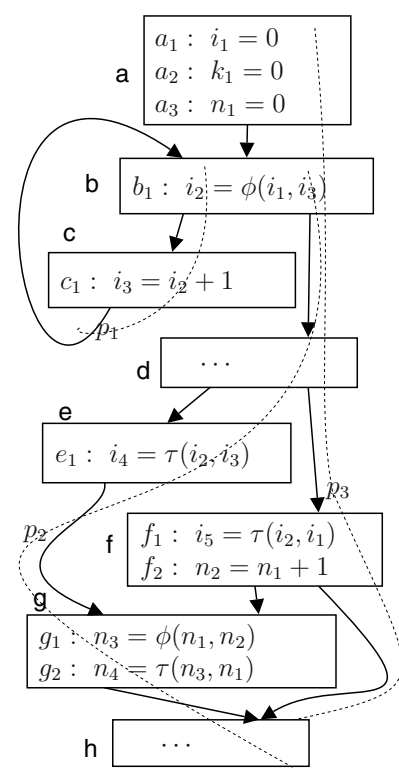

Fig. 3. HPSSA form for a program with loops (Hot paths: $p_{1}: \mathrm{bc} ; p_{2}:$ bdegh; $p_{3}:$ abdfh)

HPSSA form of a program with a loop. Notice how the variable $i_{3}$ becomes the hot reaching definition at the basic block $e$, even though $i_{3}$ reaches the node $e$ along a path that contains a backedge (as c-b is a backedge, $\mathrm{c}-\mathrm{b}-\mathrm{d}-\mathrm{e}$ is not $\mathrm{a}$ segment of any acyclic path).

In this paper, we only assume reducible flow-graphs; we also assume the existence of a loop-preheader node (leading to the loop-header) for each loop in the program.

\section{Thermal Properties of a Program}

In this section, we establish a few terms and notations that we use in the rest of the paper.

\subsection{Thermal States of Program Entities}

Definition 1. Hot/Cold Paths: A program path $p: n_{1} \rightsquigarrow n_{2}$ is said to be hot (cold) if the sequence of edges from node $n_{1}$ to $n_{2}$ appears (does not appear) in any profiled path that occurs frequently in the program profile.

The above definition has been intentionally left slightly ambiguous to make it general enough to encompass various profiling and hot path selection schemes. The phrase "profiled path" implies any sequence of basic-blocks that is collected 
by a control-flow profiler; for instance, the "profiled path" is an edge for an edge profiler, an acyclic path for a Ball-Larus path profiler, and a path spanning multiple loop iterations for a k-iteration profiler [17/20. In this paper (and our implementation), a "profiled path" refers to intraprocedural acyclic paths, profiled using a Ball-Larus profiler. The qualifier "frequently" in the above definition depends on the hot path selection scheme: we may select hot paths by a threshold frequency, or pick a finite number of the most commonly executed paths from each procedure.

Definition 2. Temperature $(\theta)$ of a node (edge) is defined as:

- hot: if the node (edge) is present on a hot path;

- cold: if the node (edge) is not present on any hot path.

A backedge $b$ in a flow-graph is marked hot if, either of the dummy edges, $\delta_{\text {start }}$ to a loop-header $h$ or $\delta_{\text {end }}$ from a loop-tail $t$, is hot 1 ; this is understandable, as any control-flow through a dummy edge reported by the Ball-Larus profiler indicates a control-flow through the corresponding backedge in the program flow-graph.

We will use the notation $\theta(n)$ to denote the temperature (hot/cold) of a program entity (nodes, edges or paths). The predicates $\theta_{h}(n) / \theta_{c}(n)$ denote that the entity $n$ is hot/cold.

For example, in Figure 1, all the nodes and edges are hot; the path $c \rightarrow d \rightarrow$ $f \rightarrow g$ is hot (through the path $p_{2}$ ) while the path $e \rightarrow f \rightarrow g$ is cold.

\section{Definition 3. Hot/Cold Reaching Definitions and Definition Chains}

$A$ definition $\delta$ at a basic-block $n_{1}$ is said to reach a respective use at a basic-block $n_{2}$ hot if there exists a hot path from $n_{1}$ to $n_{2}$, and $\delta$ is not killed along that path. $A$ definition $\delta$ at a basic-block $n_{1}$ is said to reach a respective use at a basic-block $n_{2}$ cold if there does not exist a hot path from $n_{1}$ to $n_{2}$, and $\delta$ is not killed at least along one cold path from $n_{1}$ to $n_{2}$.

Consider Figure 1 treating a $\phi$-function not as a definition, but as a label to the set of definitions in its argument set, we can see that though the meet-over-allpaths reaching definition set at $g_{3}$ is $\left\{x_{18}, x_{17}, x_{7}\right\}$, the definition $x_{18}$ does not reach it via any hot path. So, $x_{18}$ is a cold reaching definition at $g_{3}$, while $x_{7}$ and $x_{17}$ are the hot reaching definitions (reaching the node $g$ via the paths $p_{1}$ and $\left.p_{2}\right)$. In the SSA form, the $\phi$-functions can be seen as creating a definition chain, that is broken only by a non- $\phi$ definition: $x_{7} \rightarrow x_{9}$ and $x_{17} \rightarrow x_{9}$ are the hot reaching definition chains at $g_{3}$, while $x_{18} \rightarrow x_{9}$ is a cold reaching definition chain. In the HPSSA form, the $\tau$-functions "kill" the cold definition chains: for example, in Figure 2, $x_{18} \rightarrow x_{9}$ no longer reaches $g_{3}$ as it is killed by $g_{1}$.

${ }^{1}$ The Ball-Larus profiler converts a flow-graph with cycles into a directed acyclic graph (DAG) by adding dummy edges, $\delta_{\text {start }} / \delta_{\text {end }}$, to and from the backedge source/target (respectively) for each loop in the program [2]. 
The $\tau$-function can be seen as a conditional $\phi$-function: $\tau\left(x_{0}, x_{1}, \ldots, x_{n}\right)= \begin{cases}\phi\left(x_{0}\right) & \text { safe interpretation } \\ \phi\left(x_{1}, \ldots, x_{n}\right) & \text { speculative interpretation }\end{cases}$

If a program is in the Hot Path SSA form, then,

- each use of a variable is reachable by a single definition;

- if the safe interpretation of the $\tau$-function is used, each use of a variable is reachable by the meet-over-all-paths reaching definition chains;

- if the speculative interpretation of the $\tau$-function is used, each use of a variable in a hot basic-block is reachable only by the meet-over-hot-paths reaching definition chains (or the meet-over-all-paths reaching definition chains, if the use is not reachable from any meet-over-hot-paths reaching definition chain).

With the speculative interpretation, the set of reaching definition chains at even a cold basic-block might be smaller than that corresponding to the meetover-all-paths, as some of the definition chains may be "killed" by $\tau$-functions on their way to the cold node.

Each speculative argument $x_{i}$ in a $\tau$-function is mapped to the set of hot profile paths along which the definition corresponding to $x_{i}$ is reached. In Figure 2, for the variable $x$ in $g_{1}$, the $\tau$-function allocates the parameter $x_{7}$ corresponding to the path $p_{1}$, and the parameter $x_{17}$ for the path $p_{2}$. However, for the variable $y$ at $g_{2}$, it allocates only one parameter, $y_{4}$, corresponding to both $p_{1}$ and $p_{2}$ as the same definition (from statement $a_{2}$ ) reaches it along both the paths.

The HPSSA form honours the constraint imposed by the SSA form: each use is reachable by a single definition - encouraging the development of speculative extensions of existing SSA-based algorithms on the HPSSA form.

\section{Exiting the HPSSA form}

Exiting the HPSSA form is extremely simple - a $\tau$-statement is replaced by a copy statement from the safe-argument to the defined variable:

$$
x_{\text {out }}=\tau\left(x_{0}, x_{1}, \ldots, x_{n}\right) \quad \longmapsto \quad x_{\text {out }}=x_{0}
$$

This puts the program in the SSA form; one can then use a standard out-of-SSA algorithm to exit the SSA form.

\section{Constructing the HPSSA Form}

In this this section, we discuss the construction of the HPSSA form. The original program (not in SSA form) is transformed into HPSSA form in four steps:

- Insert $\phi$-statements: The classic algorithm for construction of the minimal SSA form [8] places $\phi$-statements at the iterated dominance frontier of each definition in the program. A node $v$ is said to be in the dominance frontier of another node $u$ iff $u$ does not dominate $v$ while a predecessor of $v$ is dominated by $u$. 
- Insert $\tau$-statements: For each variable $x$, we identify program points that necessitate a $\tau$-function, and, at all such points, insert a definition of the form $x=\tau(x)$ (discussed in detail in section 5.1).

- Variable renaming: The definitive variable renaming algorithm 8 uses a variable stack to propagate reaching definitions by traversing the basic-blocks over the dominator tree. The correctness of our algorithm requires a depthfirst traversal over the dominator tree. Note that this phase also renames the sole argument in the inserted $\tau$-functions to the variable version corresponding to the meet-over-all-paths "safe" reaching definition.

- Allocation of the $\tau$-function arguments: Finally, we allocate the speculative arguments to the $\tau$-functions in correspondence to the hot reaching definition chains (discussed in detail in section 5.2).

Note that after step 3, the program is in SSA form, and after step 4, it is in HPSSA form. We have intentionally kept the phases for building the SSA form (steps 1 and 3) clearly distinct from the steps required for constructing the HPSSA form (steps 2 and 4) to apprise the essentials of the HPSSA construction algorithm. It will be apparent that the phases need not be separate — some of them can be combined in an efficient implementation.

\subsection{Thermal Frontiers: Placing $\tau$-functions}

We call definitions due to $\phi$ and $\tau$ - functions as pseudo definitions, differentiating them from other concrete definitions; the corresponding statements are called pseudo/concrete statements. We define the set of visible definitions in the basicblock $u$ as the last definition of each variable in the block: these definitions are the only ones that are "seen" by the basic-blocks reachable from $u$. In the following discussion, a reaching definition would refer to only concrete definitions; pseudo reaching definitions can be seen as the set of concrete definitions that were "merged" due to a $\phi$ - or a $\tau$-function.

Each definition $x:=\ldots$ in the program can potentially lead to the insertion of a $\tau$-statement for variable $x$. In a basic-block, a $\tau$-statement is inserted after all the $\phi$-statements (if any), before any of the concrete statements.

The $\phi$-functions act as definition mergers — "merging" multiple definitions into a single one. Comparably, the $\tau$-functions act as definition filters - separating hot definitions from cold ones, which were merged by previously occurring $\phi$-functions. Hence, a node $n$ will need a $\tau$-function for a variable $v$ if, and only if, both a hot and a cold reaching definition for the variable $v$ arrive at $n$.

The minimal SSA construction algorithm uses an exquisite structure - the Dominance Frontier — to insert the $\phi$-statements. To build the HPSSA form, we identified a similar structure to place the $\tau$-statements: the Thermal Frontier.

Definition 4. Thermal Frontier: A node $v$ is said to be in the Thermal Frontier (TF) of a reaching definition $d$, where $d$ is defined at a node $u,(v \in T F(u, d))$, iff the node $v$ is also exposed to a reaching definition $d^{\prime}$, defined at a node $w$ ( $w$ not dominated by $u)$, such that $\theta(u \rightsquigarrow v) \neq \theta(w \rightsquigarrow v)$. Also, $v$ must be the first node in the paths $u \rightsquigarrow v$ and $w \rightsquigarrow v$ that satisfies the above properties. 
Stated informally, a node $v$ is in the thermal frontier of a hot/cold reaching definition $d$ (defined at $u$ ), if $v$ is also reachable by a different cold/hot (respectively) definition $d^{\prime}$ (defined at $w$ ), while being the first node along $u \rightsquigarrow v$ and $w \rightsquigarrow v$ to satisfy the conditions.

Unlike Dominance Frontiers, Thermal Frontiers need not be join nodes. For example, in Figure 2, node $g \in T F\left(b, x_{7}\right)$ as $x_{7}$ is a hot reaching definition (along $p_{1}$ ) and $g$ is also reachable by the cold reaching definition $x_{18}$.

It is apparent that $\tau$-functions for a definition $d$ at a node $u$ will be needed at the iterated $\mathrm{TF}(\mathrm{u}, \mathrm{d})$. We define the Iterated Thermal Frontier in exactly the same way as iterated join and iterated dominance frontier were defined by Cytron et al. [8].

Definition 5. Let $\gamma_{x}(u)$ return the visible definition of the variable $x$ in the basic-block $u$; then, for a set of nodes $\kappa$, the Iterated Thermal Frontier (ITF) is the limit of the increasing sequence of sets of basic-blocks:

$$
\begin{aligned}
& T F^{x}(\kappa)=\bigcup_{u \in \kappa} T F\left(u, \gamma_{x}(u)\right) \\
& T F_{1}^{x}=T F^{x}(\kappa) \\
& T F_{i+1}^{x}=T F^{x}\left(\kappa \cup T F_{i}^{x}\right) \\
& I T F^{x}=T F_{\infty}^{x} \text {, where } T F_{\infty}^{x} \text { refers to the fixpoint, i.e. when } T F_{i}^{x}=T F_{i+1}^{x}
\end{aligned}
$$

However, as the $\phi$-statements are inserted by a prior phase, placing the $\tau$ functions does not require fixpoint computation: a simple topological traversal over the CFG nodes suffices. Fixpoint computation is generally required if dataflow information can change after propagating through a backedge. While placing the $\tau$-functions, if a $\tau$-statement for a variable $x$ is inserted in the header $h$ of a loop due to a definition in the loop body (the only case that requires fixpoint computation), then, the loop-header $h$ is sure to contain a $\phi$-statement (as no node in the loop-body can dominate $h$ ). Hence, if the CFG nodes are processed in the topologial order, insertion of $\tau$-functions at the required nodes due to the definition of the variable $x$ at $h$ would have already happened.

Theorem 1. For a set of visible definitions of a variable $x$ at a set of nodes $\kappa, \tau$-statements would be required at the Iterated Thermal Frontier $I T F^{x}$ for variable $x$.

The following lemma states the necessary condition for computing the set of Thermal Frontiers.

Lemma 1. A node $n \in T F\left(u, d^{x}\right)$ for a definition $d^{x}$ (of a variable $x$ ) if

- Condition I: $n$ is the junction of a hot and a cold path, i.e., paths at different temperatures meet at this node;

- Condition II: $n$ is reachable by at least two different definitions of the variable $x$.

Proof. If condition I fails, a $\tau$-function is unnecessary as $n$ can then be reachable by only hot or only cold definitions of $x$. If condition II fails, a $\tau$-function is again unnecessary as the node is then dominated by a definition of $x$. 
However, note that the above lemma is not a sufficient condition: a node $v \notin$ $T F\left(u, d^{x}\right)$ if the same definition $d^{x}$ reaches $v$ via both a hot and cold path (satisfying condition I), while $v$ is also reachable by a different hot definition (of $x$ ), $d^{\prime}$, along a separate hot path (satisfying condition II). Hence, the above lemma may identify spurious Thermal Frontiers: our HPSSA algorithm inserts $\tau$-function templates at all points identified by the lemma, leaving the task of weeding out unnecessary $\tau$-statements to the $\tau$-argument allocation phase (section 5.2). In the rest of the discussion, we denote the set of Thermal Frontiers computed according to Lemma 1 as $T F(u, d)$, and denote the ideal set of Thermal Frontiers (as defined in Definition 4) as $T F_{\text {ideal }}(u, d)$.

Let us now sketch an algorithm for computing the Thermal Frontier of a node: we first identify certain nodes that are "junctions" of hot and cold paths (we call them Caloric Connectors), and thus, satisfy the first condition of Lemma 1, we then identify a scheme for satisfying the second condition.

\section{Caloric Connector}

Definition 6. Caloric Connector $(C C)$ : A node $n_{c c} \in C C$ if, for distinct nodes $n$ and $n^{\prime}\left(n \neq n^{\prime}\right)$, there exist paths $n \rightsquigarrow n_{c c}, n^{\prime} \rightsquigarrow n_{c c}$ such that $\theta\left(n \rightsquigarrow n_{c c}\right) \neq$ $\theta\left(n^{\prime} \rightsquigarrow n_{c c}\right)$, and for all nodes $n^{\prime \prime} \in\left(N\left(n \rightsquigarrow n_{c c}\right) \cap N\left(n^{\prime} \rightsquigarrow n_{c c}\right)\right)-\left\{n_{c c}\right\}$, $n^{\prime \prime} \notin C C$.

In other words, a node $n_{c c}$ is a Caloric Connector in a given graph (for a given set of hot paths) if there exist distinct nodes $n$ and $n^{\prime}$, such that $n$ and $n^{\prime}$ can reach $n_{c c}$ through paths having different temperatures, and $n_{c c}$ is the first common node in $n \rightsquigarrow n_{c c}$ and $n^{\prime} \rightsquigarrow n_{c c}$ satisfying these properties.

Consider Figure 1, the node $g$ is a Caloric Connector as the path $d \rightarrow f \rightarrow g$ is hot while $e \rightarrow f \rightarrow g$ is cold, while both the "predecessor" paths $(d \rightarrow f$ and $e \rightarrow f)$ are hot.

Lemma 2. A hot acyclic path $t \rightsquigarrow u$ extended by a forward edge $u \rightarrow v$ forms a cold path $t \rightsquigarrow u \rightarrow v$ if, for some incubation node $s$, there exists a set of buddy paths $B \in B_{\text {Bddy }} \operatorname{Set}_{s}(u)$ among the paths at $u$, such that none of the buddies $\sigma \in B$ traverse the edge $u \rightarrow v$.

Lemma 3. If an acyclic path $t \rightsquigarrow u \rightarrow v$ is cold, then, either

$-t \rightsquigarrow u$ is cold, or

- $s \rightsquigarrow t \rightsquigarrow u$ is hot, and $\exists B \in B u d d y \operatorname{Set}_{s}(u)$, such that none of the buddies $\sigma \in B$ traverses $u \rightarrow v$ (where $s$ is the incubation node for $s \rightsquigarrow t \rightsquigarrow u$ ).

The intuition for the above lemmas is as follows: Each set of buddies at $u$, $B_{i} \in B_{u d d y} \operatorname{Set}_{s}(u)$, correspond to a unique sequence of edges $(s \rightsquigarrow u)_{i}$ from $s$ to $u$, distinct from that of any other buddy set $B_{j} \in \operatorname{Buddy}_{\operatorname{Set}}(u), B_{i} \neq B_{j}$. If no hot path $p \in B_{i}$ selects the edge $u \rightarrow v$, that particular sequence of edges $(s \rightsquigarrow u)_{i} \rightarrow v$ is surely missing among the hot paths reaching $v$. This implies that the path $(s \rightsquigarrow u)_{i} \rightarrow v$ is cold. We omit the formal proofs for want of space. 


\section{Algorithm 1. Computing the set of Caloric Connectors}

Traverse each node $v$ in the graph (in the topological order) in the following manner:

1. Initialize hasAColdPath and hasAHotPath to false.

2. For all edges $e: u \rightarrow v$,

- if $\theta_{c}(u \rightarrow v)$, set hasAColdPath = true;

- if $\theta_{h}(u \rightarrow v)$,

(a) Set hasAHotPath = true;

(b) If $e$ is not a backedge, and if, $\exists B \in \operatorname{BuddySet}_{s}(u)$ (for some incubation node $s$ ) such that $B$ does not intersect Paths $(u \rightarrow v)$, set has AColdPath = true.

3. If both hasAColdPath and hasAHotPath are true, add $v$ to the set of Caloric Connectors.

The algorithm for computing the set of Caloric Connectors (Algorithm 1) is targeted at identifying if both a hot and a cold path can reach a node. Iterating through all nodes in the CFG in topological order, for each node $u$, the algorithm examines the temperature of each outgoing edge $u \rightarrow v$. It decides on the existence of a hot and/or a cold path at $v$ in accordance to Lemma 2 and 3, and sets the flags hasHotPath and hasColdPath accordingly. A node $v$ is marked as a Caloric Connector if it has both a hot and a cold path reaching it.

Computing Thermal Frontiers. For a concrete definition $d$ and a basic-block $v \in T F(u, d)$, the second condition of Lemma 1 is satisfied if $v$ is in the dominance frontier of $\mathrm{u}$ (the node $v$ is then also exposed to a different definition $d^{\prime}$ at a node $w$ that is not dominated by $u$ ).

The case for pseudo definitions is slightly different: We

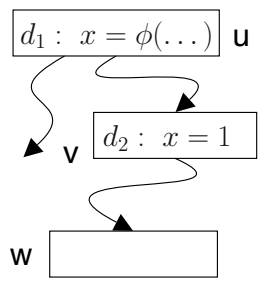

Fig. 4. Violation of condition II of Lemma 1 ideate a $\phi$-statement $x_{3}=\phi\left(x_{1}, x_{2}\right)$ not as a single definition, but as a set of concrete definitions $\left\{x_{3}=x_{1}, x_{3}=x_{2}\right\}$ being propagated to all the outgoing paths from the definitionsite; we also envision the $\tau$-statements similarly, but with only the speculative argument:2. As all paths from a pseudo definition $d$, defined at a node $u$, are now ideated as carrying this set of definitions (instead of just $d$ ), the first Caloric Connector $\left(n_{c c}\right)$ on each outgoing path from $u$, called the Closest Caloric Connectors of $u(\mathrm{CCC}(\mathrm{u}))$, satisfies Lemma 1 - provided the pseudo-definition $d$ actually reaches $n_{c c}$. Figure 4 illustrates this case when $d$ does not reach $n_{c c}$ : Let $w \in C C C(u)$; however, $w \notin T F\left(u, d_{1}\right)$ as the pseudo-definition $d_{1}$ is "killed" by the concrete definition $d_{2}$ at $v$, making $d_{2}$ the dominating definition for $w-$ violating condition II of Lemma 1

Algorithm 2 outlines our algorithm for inserting $\tau$-nodes.

\footnotetext{
${ }^{2}$ In the HPSSA construction algorithm, the hot definitions are "percolated" through the $\phi$ and $\tau$ statements as the percolated definitions may appear as arguments to future $\tau$-statements.
} 


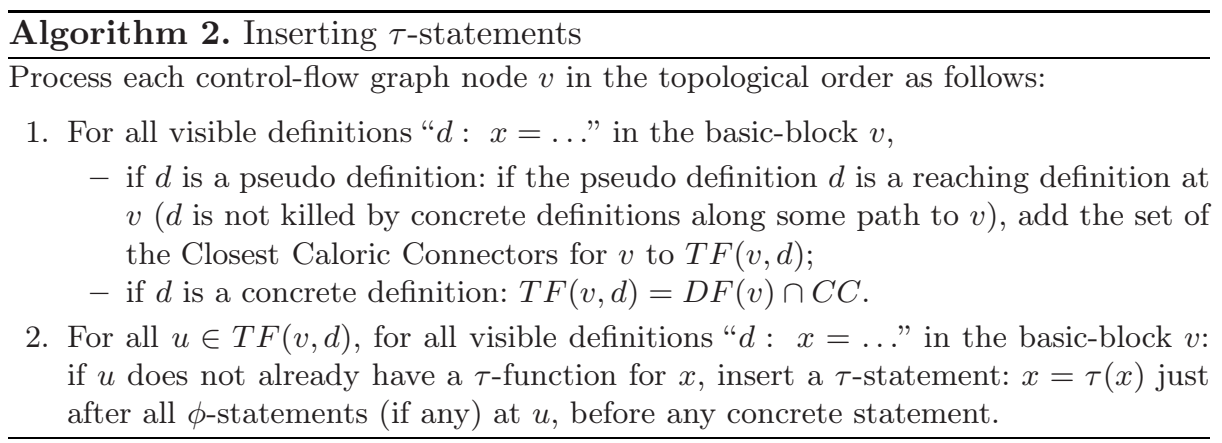

\subsection{Allocating $\tau$-function Arguments}

Before delving into the details of the algorithm, we take a slight digression into a deeper understanding of the $\phi$ and $\tau$ statements. We view a pseudo definition not as a new definition - but as a label to an existing set of definitions, namely, the definitions corresponding to its argument set. So, when we talk of reaching definitions in this section, we would refer to all definitions (pseudo and concrete) that are not killed by a concrete definition; we do not allow pseudo definitions to kill an existing set of definitions. For example, in Figure 2 we would say that the definitions for $x_{9}, x_{17}$, and $x_{7}$ are the set of hot definitions that reach $g$; we call this set as the set of active definitions at $g$. In the SSA form, as each definition corresponds to a unique version of the variable, we use the terms definition and variable version interchangeably.

The algorithm, in essence, computes the path-sensitive active reaching definitions at each node $u$ containing a $\tau$-function. The hot reaching definitions (variable versions) stand as arguments in the $\tau$-functions at $u$, each definition mapped to the set of hot paths along which it reaches $u$. A definition $x_{i}$ that reaches $u$ along the set of hot-paths $\xi_{i}$ can be used as a parameter for a $\tau$-function only if the following conditions are satisfied:

- if $x_{i}$ is a concrete reaching definition: $x_{i}$ can only be used as a parameter if $\xi_{i} \neq \emptyset$, i.e., it does reach $u$ along a hot path;

- if $x_{i}$ is a pseudo reaching definition: As discussed above, pseudo definitions are just labels to a set of concrete definitions. Even if $\xi_{i} \neq \emptyset$, not all concrete definitions contained 3 in $x_{i}$ may be reaching $u$ : In Figure 2 , the pseudodefinition $x_{9}$ reaches $g_{1}$ along the hot paths $\xi_{i}=\left\{p_{1}, p_{2}\right\}$, i.e. $\xi_{i} \neq \emptyset$. However, if $x_{9}$ is used as parameter for the $\tau$-function at $g_{1}$, it would invariably mean the inclusion of the definition $x_{18}$, which is not a hot reaching definition at $g$. Hence, a pseudo-definition can be used as an argument for some set of hot paths $\xi$ if, and only if, all the concrete reaching definitions that it merges reaches $u$ along $\xi$. This condition can be ensured by checking if all

\footnotetext{
${ }^{3}$ A definition for $x_{i}$ is contained in a $\phi$-definition if the $\phi$-function argument-list either includes $x_{i}$, or includes a variable-version $x_{j}$ such that $x_{i}$ is contained in the definition for $x_{j}$; for the $\tau$-functions, we only consider the speculative argument-list.
} 
the contained concrete definitions for $x_{i}$ are available as active definitions at $u$ for the set of paths $\xi$.

Allowing definitions corresponding to pseudo-definitions in the $\tau$-function argument list requires tracking of both pseudo and concrete definitions (which might appear along intersecting set of paths), while ensuring that a pseudo definition never kills a concrete definition, even along the same path. For the sake of simplicity, we abandon any further discussion on the same: in the following discussion, we ignore all pseudo definitions and maintain only the concrete definitions as active definitions (except if a pseudo-definition occurs as the only available reaching definition, or if a pseudo-definition is propagated along a backedge). As pseudo-definition "labels" to a set of merged definitions can no longer appear in the $\tau$-function argument lists, the implication of ignoring the pseudo definitions is a larger argument list for the $\tau$-functions.

Instead of performing an expensive classical path-sensitive dataflow analysis, we designed an algorithm very similar to the variable renaming phase of SSA construction [8] - using a variable stack to maintain the active definitions (or renamed variables) reaching each node. Our algorithm is defined as a recursive procedure running over the dominator tree of the control-flow graph. The variable stack maintains the set of active reaching definitions $\left(x_{i}\right)$, along with the set of hot paths $\left(\xi_{i}\right)$ that carry the definitions to the current node 4 . Our algorithm is more efficient than context-tupled classical path-sensitive dataflow analysis as it does not require storing of path-sensitive dataflow information at each basic-block.

Let $P$ be the set of profiled acyclic path identifiers, and DefPaths be the set of $P$. A frame in the variable stack is a map [DefPaths $\mapsto$ Version], where Version is the renamed version of a variable; a frame can be seen as a set containing pairs $\left\{\left[\xi_{1}, x_{1}\right],\left[\xi_{2}, x_{2}\right], \ldots,\left[\xi_{n}, x_{n}\right]\right\}$, where $\xi_{i} \in$ Def Paths. A variable stack $\operatorname{Var} S t a c k_{x}$ is a stack of frames for the base variable $x$.

VarStack supports the following operations: push ( $\xi_{i}$ :DefPaths, $x_{i}$ :Version, $\mathrm{u}$ :Basic-block) pushes a new frame with the association $\left[\xi_{i}, x_{i}\right]$ on $\operatorname{Var} S t a c k_{x}$; pop (u:Basic-block) pops off all frames that were pushed in the basic-block $u$; and top() returns the topmost frame on the stack.

A Frame in VarStack supports the following operations: get ( $\xi$ :DefPaths) returns the version associated with $\xi$ in the map; accumulate ( $\xi_{i}$ :DefPaths, $x_{i}$ :Version) accumulates definitions: if a pair $\left[\xi_{j}, x_{i}\right] \in$ Frame, replace $\left[\xi_{j}, x_{i}\right]$ by $\left[\xi_{j} \cup \xi_{i}, x_{i}\right]$, else add a new association $\left[\xi_{i}, x_{i}\right]$ to the frame.

The top of the variable stack contains the set of active definitions - definitions that can be used to allocate arguments to the $\tau$-functions in the current basicblock. The algorithm traverses the control-flow graph recursively in a depth-first order over the dominator tree (as does the variable renaming phase for SSA construction); the set of dominatee 5 are traversed in the topological order of

\footnotetext{
${ }^{4}$ The updates to $\xi_{i}$ is done lazily; so a certain points, they may contain more paths than the actual set of hot reaching paths.

${ }^{5}$ The children of a node $n$ in the dominator tree are the dominatees of $n$.
} 
the nodes in the control-flow graph: the order is important to ensure that when a basic-block is processed, the definitions from all its incoming paths reach it. The active definitions are propagated via VarStack from a parent node to its children in the dominator tree; for a join node $u$, the active definitions are accumulated (by a similar operation as accumulate ( $\xi_{i}$ :DefPaths, $x_{i}$ :Version) for a frame) in a Definition Accumulator $\Omega_{x}(u)$ from its predecessors in the CFG - it is loaded up on VarStack when the node $u$ is processed.

The $\tau$-allocation algorithm is sketched in Algorithm 3. Let us describe the algorithm via an example (Figure 5) for the flow-graph in Figure 2

Let the basic-blocks be processed in the order $a, b, c, d, e, f, g, h, i$.

The basic-block $a$ is processed foremost: the algorithm (Step 3(c)) pushes the definition $x_{3}$ on $\operatorname{VarStack}_{x}$ (Figure (a)), and then recurses on the children of $a$ in the dominator tree, namely $b, c$ and $f$ (Step 5). At the node $b$, the algorithm (Step 3(c)) pushes the definition $x_{7}$ on the stack; its successor node, $f$, turns out to be a join node: hence, the algorithm (Step 4) accumulates the definitions in the topmost frame of the stack into the (currently empty) definition accumulator $\Omega_{x}(f)$ (Figure (b)). As $b$ has no children in the dominator tree, the algorithm (Step 6) retraces the recursive path to node $a$, popping off the definition pushed by $b$ in the process. The variable stack and the recursion stack $\left(S_{R}\right)$ now again resemble that in Figure (a).

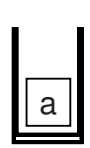

$S_{R}$

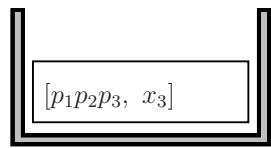

$\operatorname{VarStack}_{x}$

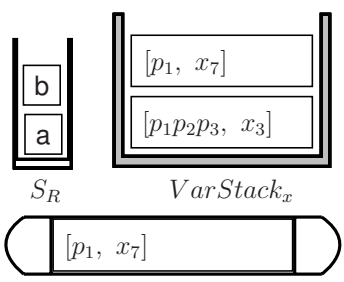

$\Omega_{x}(f)$

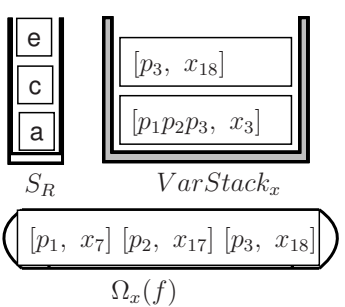

(a) After node $a$ is pro- (b) After node $b$ is processed cessed
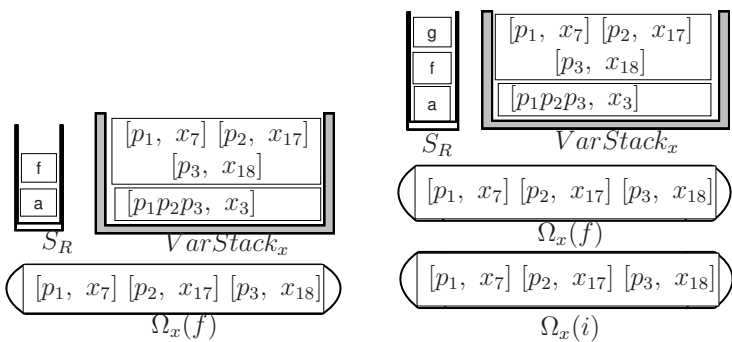

(c) After node $e$ is processed

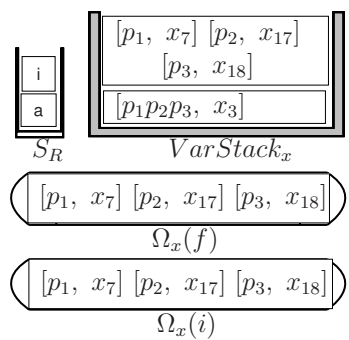

(d) After node $f$ is pro- (e) After node $g$ is processed (f) After node $i$ is processed cessed

Fig. 5. Steps in the execution of the $\tau$-argument allocation algorithm 
Algorithm 3. A sketch of the $\tau$-function argument allocation algorithm

Process a basic-block $u$ in the following manner:

1. Push the Definition Accumulator $\Omega(u)$ on VarStack (if $\Omega(u)$ exists).

2. If $u$ is the incubation node for a set of hot paths, for all base-variables $x$ which do not have a $\phi$-definition appearing in the basic-block $u$, push a frame $\left\langle\xi_{i}, x_{i}\right\rangle$, where $\xi_{i}$ is the set of all paths that incubate from $u$, and $x_{i}$ is the meet-over-all paths reaching definition (variable-version) for $x$ at $u$.

3. Process each statement stm in the basic-block:

(a) If $s t m$ is a $\phi$-statement: if $u$ is a loop-header and the dummy profile edge $t \rightarrow$ $\delta_{\text {end }}$ is hot (where $\delta_{\text {end }}$ denotes the dummy-end node for a Ball-Larus profiler, and $t$ is the corresponding loop-tail), accumulate $\left\langle\xi_{i}, x_{i}\right\rangle$ at the topmost frame of $\operatorname{VarStack}$, where

i. $\xi_{i}$ is the set of all paths that incubate from $u$, and

ii. $x_{i}$ is the $\phi$-statement argument corresponding to the backedge $t \rightarrow u$.

(b) If $s t m$ is a $\tau$-statement:

i. Create a set $\mathrm{C}$ of candidate definitions from the definitions in $\operatorname{VarStack.top}()$ for each incubation node $s$ : add $\left\langle\xi_{i}, x_{i}\right\rangle$ to $\mathrm{C}$ iff $\left(\right.$ Path $\left._{s}(u) \cap \xi_{i}\right) \neq \emptyset$;

ii. If there exists at least one $x_{i} \in C$ such that its variable-version differs from the safe argument $x_{0}$, add arguments to the $\tau$-function for each $x_{i}$, mapping the respective variable position to $\xi_{i}$; otherwise, replace the $\tau$ function with a simple copy statement: $x_{\text {out }}=x_{0}$.

(c) Update VarStack to include new definitions in the basic-block $u$ :

- Concrete definition: Push the definition as a new frame associating it with $\operatorname{Paths}(u)$;

- Pseudo definition: Ignore.

4. Save the active definitions in $\Omega$ of the (forward) successors (if successor is a join node): for each forward (ignore backedges) successor edge $u \rightarrow v$, if $v$ is a join node, for each $\left\langle\xi_{i}, x_{i}\right\rangle \in \operatorname{VarStack.top}()$ such that $\left(\xi_{i} \cap \operatorname{Paths}(u \rightarrow v)\right) \neq \emptyset$, accumulate $\left\langle\xi_{i} \cap\right.$ Paths $\left.(u \rightarrow v), x_{i}\right\rangle$ in $\Omega_{x}$.

5. Recurse on the children of $u$ in the dominator tree in accordance to their topological order in the control flow graph.

6. Pop off all frames pushed by $u$ from VarStack.

The nodes $c, d$, and $e$ are processed similarly; Figure (c) shows the state of the data-structures just after node $e$ is processed. After handling $e$, the recursion is unwound to node $a$.

The algorithm then picks the node $f$ : it first pushes the definition accumulator of $f, \Omega_{x}(f)$, on the variable stack (Step 1); on encountering the $\phi$-definition for $x_{9}$, it simply ignores the same (Step 3(c)). Finally, it recurses on the immediate dominatees of $f$, viz. $g$ and $h$ (Step 5).

The node $g$ is processed next: on encountering the $\tau$-definition for $x_{11}$, the algorithm (Step 3(b)) attempts to allocate arguments for the same: Examining the active definitions (top of the variable stack), the algorithm attempts to assemble the candidate set $C$ - a subset of definitions from the topmost frame of $\operatorname{VarStack}_{x}$ that, together, can map to all the hot paths passing through $g$. 
The set of active definitions at $g$ turn out to be $\left\{\left[p_{1}, x_{7}\right],\left[p_{2}, x_{17}\right],\left[p_{3}, x_{18}\right]\right\}$. To be added to $C$, the path-component in the definition pairs must intersect with $\operatorname{Paths}(g)=\left\{p_{1}, p_{2}\right\} ;\left[p_{1}, x_{7}\right]$ and $\left[p_{2}, x_{17}\right]$ satisfy the condition, while $\left[p_{3}, x_{18}\right]$ does not. Notice how the cold definitions are pruned are from the possible set of definitions to be added as arguments to the $\tau$-function.

As the variable versions in the set $\mathrm{C}$ differ from that of the safe argument, we allocate arguments to the $\tau$-function from $\mathrm{C}$.

$$
x_{11}=\tau\left(x_{9}, x_{7}\left\langle p_{1}\right\rangle, x_{17}\left\langle p_{2}\right\rangle\right)
$$

The algorithm then accumulates the active definitions in $\Omega_{x}(i)$ (Figure (e)). The nodes $h$, and then $i$ are processed in order in a similar manner.

Note that the set of candidate definitions $C$ for a $\tau$-function at a node $v$ contains the exact set of hot definitions that reach $v$. Additionally, for each pair $\left\langle\xi_{i}, x_{i}\right\rangle \in C, x_{i}$ reaches $u$ along the paths in $\xi_{i}$, and along no other hot path.

Now consider the control-flow graph with loops (Figure [3): Let us illustrate as to how the the hot reaching definition of $i_{3}$ in the block $c$ is identified as a hot reaching definition at the $\tau$-function in the node $e$ even though we use acyclic path-profiles. As the loop-path $p_{1}$ is hot, when the node $b$ is processed, the definition-pair $\left\langle p_{1} p_{2}, i_{3}\right\rangle$ is added to the top of the variable stack (being the parameter to the $\phi$-function corresponding to the backedge) by Step 3(a). When the algorithm recurses on the children of $d$ in the dominator tree, the variable stack carries the definition to the basic-block $e$ where it is recognised as an argument for the $\tau$-function along the path $p_{2}$. The Step 2 in the algorithm is required to carry the meet-over-all-paths definition $n_{1}$ from the node $a$ to the node $g$, as there does not exist any acyclic hot path from $a$ to $g$.

\section{Speculative Sparse Conditional Constant Propagation}

We have implemented the analysis phase of a novel optimization - the Speculative Sparse Conditional Constant Propagation (SSCP) on the HPSSA form. This optimization expands the scope of the SCP 21] algorithm - allowing it to identify speculative constants (expressions that are highly likely to be constants) - along with the conventional "safe" constants (expressions that are guaranteed to be constants).

This section is more than a description of a new analysis - through this novel analysis, we essentially aim to demonstrate how new speculative optimizations can be developed on the HPSSA form by simple extensions of existing "safe" SSA-based optimizations.

The SSCP algorithm operates on a four level lattice (Figure 6 shows the SSCP lattice for integers): the conventional constant propagation lattice is extended by another layer — that of speculative constants (indicated by the constants superscripted with 's'). The speculative constants can be seen as constant values with exactly the same properties as that of ordinary constants - just marked "speculative" - indicating that they are predicted values, not guaranteed to hold under all executions. 
The transfer functions of all existing operations (including that of the $\phi$-function) hold as in SCP, except for the fact that if any operand in an expression turns out to be a speculative constant, the result of the operation, if a constant, would be a speculative constant carrying the respective constant value. For example, $2+$ $3^{s}$ would render the speculative constant $5^{s}$.

The transfer function for the $\tau$-functions is defined as follows

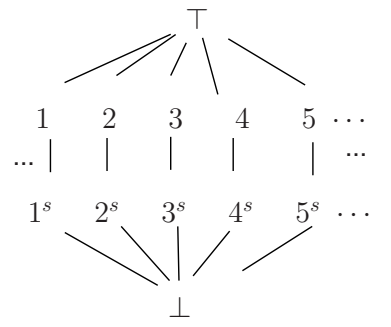

Fig. 6. The SSCP Lattice (the constants superscripted by 's' are the speculative constants)

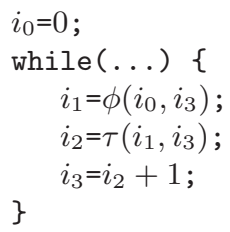

Fig. 7. A case that requires meet with its old value in the $\tau$-statement transfer function for SSCP (where $\square$ is the meet operator): If the meet of all the arguments does not produce $\perp$ (not-constant), the transfer function resembles the transfer function for the $\phi$-functions. Even if the meet of all the arguments turns out to be $\perp$, there might still be the chance of the expression being identified as a speculative constant: let $\beta=x_{1} \sqcap x_{2} \ldots \sqcap x_{n}$. The transfer function attempts to return $\beta$, if $\beta$ is $\perp, T$ or a speculative constant; if $\beta$ is a "safe" constant, $\beta$ moves in the lattice to $(\beta)^{s}$, the corresponding speculative constant. Formally, the transfer function for $\tau\left(x_{0}, x 1, \ldots, x_{n}\right)$ is given by the following (where each expression refers its abstract value in the lattice, and $\left.\beta=x_{1} \sqcap x_{2} \ldots \sqcap x_{n}\right)$ :

$$
\tau\left(x_{0}, x 1, \ldots, x_{n}\right) \sqcap \begin{cases}x_{0} \sqcap \beta & \text { if } x_{0} \sqcap \beta \neq \perp \\ \beta & \text { if } x_{0} \sqcap \beta=\perp \text { and } \beta \text { is not a safe constant } \\ (\beta)^{s} & \text { otherwise }\end{cases}
$$

The meet with the current value of the $\tau$-function is added to ensure termination by ensuring monotonicity; otherwise, code fragments resembling that in Figure 7 will never reach a fixpoint due to $i_{3}$ increasing its value in the speculative domain, and the $\tau$-function feeding the same value back to it. We omit detailed discussions on this analysis for want of space.

\section{Implementation and Experiments}

We implemented our HPSSA construction algorithm, as well as the analysis phase of the SSCP algorithm on the Scale compiler [18; we were also aided by the CIL [7] tool. We only cast scaler variables whose address has not been taken in the HPSSA form; $\tau$-functions are not introduced for the remaining variables. The SSCP algorithm implementation handles only integer variables; the implementation is interprocedural but context-insensitive; function pointers are ignored (it flags a warning, computing a possibly unsafe solution).

We tested our implementation on some programs from the SPEC2000 benchmark suite. We used a naive hot path selection criteria: all the acyclic paths executed on the train input set was considered "hot" for building the HPSSA 
Table 1. Speculative Constants discovered by the SSCP algorithm. ( ${ }^{\prime}$, indicates almost; grp, prg, \& src refer to inputs graphic, program \& source respectively).

\begin{tabular}{|c|c|c|c|c|c|c|c|c|}
\hline \multirow{2}{*}{ Program } & \multirow{2}{*}{ Inpt } & \multicolumn{2}{|c|}{ Variable Uses } & Expression Uses & \multicolumn{3}{|c|}{ Total } \\
\hline \hline 181.mcf & - & Uses & HitRt & Uses & HitRt & Hits & Misses & HitRt \\
\hline 175.vpr & - & 6938074 & 100.00 & 49665 & 100.00 & 82775 & 0 & 100.00 \\
\hline \multirow{3}{*}{ 164.gzip } & grp & 26592 & 100.00 & 8110837 & 100.00 & 15048911 & 0 & 100.00 \\
\cline { 2 - 10 } & prg & 17412 & 100.00 & 5 & 100.00 & 17417 & 0 & 100.00 \\
\cline { 2 - 9 } & src & 4721 & 99.98 & 5 & 100.00 & 4725 & 1 & 99.98 \\
\hline 197.parser & - & 165970964 & 100.00 & 340 & 97.94 & 165970861 & 443 & 100.00 \\
\hline & grp & 132106650 & $\sim 100.00$ & 938 & 76.97 & 132107372 & 216 & 100.00 \\
\cline { 2 - 9 } & prg & 100819492 & 100.00 & 6576416 & 15.67 & 101849942 & 5545966 & 94.84 \\
\cline { 2 - 9 } & src & 108134316 & $\sim 100.00$ & 5256006 & 17.94 & 109077366 & 4312956 & 96.20 \\
\hline
\end{tabular}

form. Table 1 exhibits our findings for programs run on the ref input set. The programs were run with the default parameters, i.e., no parameters were set on the command line, either for training, or for the actual run (on the ref set). We collected statistics for dynamic uses (use of a variable/expression during the actual run) for variables (Variable Uses), and for sub-expressions that could be constant-folded speculatively (Expression Uses). The uses are tabulated only for the speculative constants - uses that are likely (but not guaranteed) to be constants. We have not shown the number of "sure" constants as it would be same as that for the original SCP algorithm. We also indicate the Hit Rate (HitRt): the percentage of uses where the use of variable/expression actually agrees with the "predicted" speculative constant value.

The programs seem to enshroud plenitude opportunities for an optimizer adept at performing speculative program transformations. Most of the programs show a large number of dynamic speculative usages with good hit rates (except 256.bzip2 for the sub-expression uses; still the overall hit rate turns out high, courtesy the variable usages). A more intelligent hot path selection scheme may be able to reap more constants, though it may also have an effect on the hit-rate; we are interested in experimenting with alternative schemes in the future.

\section{Related Work}

Multitude of interesting extensions and modifications have been proposed on the SSA form. The Hashed SSA (HSSA) form [6] extends the traditional SSA form to accommodate pointer variables by introducing an explicit may modify operator $(\chi)$ and may reference operator $(\mu)$. The Array SSA [13] form captures elementlevel data flow information of array variables. The $\psi$-SSA form [19] simplifies the use of SSA-based optimizations on predicated code. Though we have not addressed aliasing and arrays in this paper, it does not seem difficult to address these issues in the HPSSA form; we may investigate such extensions via concrete implementations in the future. 
Lin et al. 14 proposed a speculative SSA form by extending speculative versions of the HSSA operators - speculative update $\left(\chi_{s}\right)$ and speculative use $\left(\mu_{s}\right)$. The speculative flag, either by use of profiling information and/or a set of heuristic rules, is turned on these operators if it is highly likely that an update or reference will be substantiated at runtime. Lin et al.'s work is orthogonal to our work as we target exposing the hot use-def chains rather than likely alias relations; both these techniques can be seamlessly combined for a more powerful speculative optimization framework.

Towards path-sensitive program optimizations, Ammons and Larus [1] proposed performing flow-analysis on a hot path graph that isolates the frequent paths. Das et al. [10] proposed a polynomial-time path-sensitive algorithm for verifying a given temporal safety property, and proved it effective by verifying the file I/O behaviour of a version of the GNU C Compiler.

Researchers have also been interested in inferring likely data-flow facts, computed over control-flow profiles. Ramalingam [16] used edge-profiles to infer the probability with which a fact holds true for the class of finite bi-distributive subset problems. Probabilistic pointer analyses 49] assign probabilities with which a points-to relation might hold at a program point. Contributions to speculative partial redundancy elimination have been made by $[1522$. Path profile based speculative PRE and PDE have been proposed by [1112. Most of these techniques use edge and node profiles which are much weaker than path-profiles used by HPSSA. Also, the HPSSA form provides a common ground for writing efficient optimizations on a sparse program representation; it scores over flow-based speculative optimizations due to the exact reason that the SSA-based algorithms score over the flow-based safe optimizations.

\section{Conclusions}

We propose a novel extension to the highly successful SSA form, and demonstrate - by an analysis algorithm for Speculative Sparse Conditional Constant Propagation - that novel speculative optimizations can be enabled on the HPSSA form by almost obvious modifications of existing SSA-based traditional optimizations. We are pondering over the design of speculative versions of other existing SSA-based traditional optimizations - Global Value Numbering [3] and Partial Redundancy Elimination [5] being our foremost targets. We are also interested in extending the HPSSA form for richer profiles like the k-iteration [17] profiles.

Acknowledgements. Subhajit Roy was supported by Doctoral Fellowship from Philips Research, India.

\section{References}

1. Ammons, G., Larus, J.R.: Improving data-flow analysis with path profiles. SIGPLAN Not. 39(4), 568-582 (2004)

2. Ball, T., Larus, J.R.: Efficient path profiling. In: International Symposium on Microarchitecture (MICRO), pp. 46-57 (1996) 
3. Briggs, P., Cooper, K.D., Taylor Simpson, L.: Value Numbering. Software: Practice and Experience (1997)

4. Chen, P.-S., Hwang, Y.-S., Ju, R.D.-C., Lee, J.K.: Interprocedural Probabilistic Pointer Analysis. IEEE Transactions on Parallel and Distributed Systems 15(10), 893-907 (2004)

5. Chow, F., Chan, S., Kennedy, R., Liu, S.-M., Lo, R., Tu, P.: A new algorithm for partial redundancy elimination based on SSA form. In: Programming Language Design and Implementation (PLDI), pp. 273-286 (1997)

6. Chow, F.C., Chan, S., Liu, S.-M., Lo, R., Streich, M.: Effective Representation of Aliases and Indirect Memory Operations in SSA Form. In: Gyimóthy, T. (ed.) CC 1996. LNCS, vol. 1060, pp. 253-267. Springer, Heidelberg (1996)

7. CIL - Infrastructure for C Program Analysis and Transformation, http://hal.cs.berkeley.edu/cil/

8. Cytron, R., Ferrante, J., Rosen, B.K., Wegman, M.N., Kenneth Zadeck, F.: Efficiently computing static single assignment form and the control dependence graph. ACM Trans. Program. Lang. Syst. 13(4), 451-490 (1991)

9. Da Silva, J., Gregory Steffan, J.: A probabilistic pointer analysis for speculative optimizations. SIGARCH Comput. Archit. News 34(5), 416-425 (2006)

10. Das, M., Lerner, S., Seigle, M.: ESP: path-sensitive program verification in polynomial time. In: Programming Language Design and Implementation (PLDI), pp. 57-68 (2002)

11. Gupta, R., Berson, D.A., Fang, J.Z.: Path Profile Guided Partial Dead Code Elimination Using Predication. In: Parallel Architectures and Compilation Techniques (PACT), p. 102. IEEE Computer Society, Washington, DC (1997)

12. Gupta, R., Berson, D.A., Fang, J.Z.: Path Profile Guided Partial Redundancy Elimination Using Speculation. In: International Conference on Computer Languages (ICCL), p. 230 (1998)

13. Knobe, K., Sarkar, V.: Array SSA form and its use in parallelization. In: Principles of Programming Languages (POPL), pp. 107-120 (1998)

14. Lin, J., Chen, T., Hsu, W.-C., Yew, P.-C., Ju, R.D.-C., Ngai, T.-F., Chan, S.: A compiler framework for speculative analysis and optimizations. In: Programming Language Design and Implementation (PLDI), pp. 289-299 (2003)

15. Horspool, R.N., Pereira, D.J., Scholz, B.: Fast Profile-Based Partial Redundancy Elimination. In: Lightfoot, D.E., Szyperski, C. (eds.) JMLC 2006. LNCS, vol. 4228, pp. 362-376. Springer, Heidelberg (2006)

16. Ramalingam, G.: Data flow frequency analysis. In: Programming Language Design and Implementation (PLDI), pp. 267-277 (1996)

17. Roy, S., Srikant, Y.N.: Profiling k-Iteration Paths: A Generalization of the BallLarus Profiling Algorithm. In: International Symposium on Code Generation and Optimization (CGO), pp. 70-80. IEEE Computer Society, Washington, DC (2009)

18. Scale: A Scalable Compiler for Analytical Experiments, http://www-ali.cs.umass.edu/Scale/

19. Stoutchinin, A., de Ferriere, F.: Efficient static single assignment form for predication. In: International Symposium on Microarchitecture (MICRO), pp. 172-181 (2001)

20. Tallam, S., Zhang, X., Gupta, R.: Extending path profiling across loop backedges and procedure boundaries. In: International Symposium on Code Generation and Optimization (CGO), pp. 251-264 (2004)

21. Wegman, M.N., Kenneth Zadeck, F.: Constant propagation with conditional branches. ACM Trans. Program. Lang. Syst. 13(2), 181-210 (1991)

22. Xue, J., Cai, Q.: A lifetime optimal algorithm for speculative PRE. ACM Trans. Archit. Code Optim. 3(2), 115-155 (2006) 